\title{
Plenitud ótica como primer síntoma en patología del espacio parafaríngeo
}

\section{Otic fullness as a first symptom in parapharyngeal space pathology}

\author{
Aina Brunet $\mathbf{G}^{1}$, $\mathbf{M}$ José Ferrer $\mathbf{R}^{1}$, Raquel Solavera $\mathbf{S}^{1}$, Iván Doménech $\mathbf{M}^{1}$, Marta Faubel $\mathbf{S}^{1}$.
}

\begin{abstract}
RESUMEN
Los tumores del espacio parafaríngeo (EP) son poco frecuentes, representando el 0,5\%-1\% de las neoplasias de cabeza y cuello. La distribución de los tumores del EP constituye: $40 \%$ tumores de glándulas salivales, seguidos de tumores neurogénicos y adenopatías. Presentamos un caso de una paciente de 52 años que acude por presentar sensación de taponamiento ótico izquierdo y molestias faríngeas de 3 meses de evolución. Tras una exploración otorrinolaringológica completa se sospecha patología del espacio parafaríngeo, que se confirma con las pruebas de imagen. Se realiza exéresis quirúrgica mediante abordaje transcervical-transparotídeo, con buena evolución posoperatoria y sin recidiva tras 1 año de seguimiento. El estudio anatomopatológico informa adenoma pleomorfo de parótida. En este trabajo se ha realizado una revisión de la etiopatogenia, diagnóstico y tratamiento de estas lesiones. Consideramos crucial realizar una exploración física otorrinolaringológica completa ante la presencia de un paciente con sintomatología inespecífica ya que el EP constituye un área anatómica difícil de explorar y que a menudo pasa desapercibida, por lo que la patología del EP representa un reto diagnóstico y terapéutico.
\end{abstract}

Palabras clave: Espacio parafaríngeo, adenoma pleomorfo, parótida, plenitud ótica.

\begin{abstract}
Parapharyngeal space (PPS) tumors are infrequent and account for $0.5 \%-1 \%$ of head and neck neoplasms. Therefore, they represent a diagnostic challenge. The distribution of PPS tumors is as follows: $40 \%$ salivary tumors, followed by neurogenic tumors and adenopathies. We report a case of a 50 year old woman that presented with a 3-month history of otic fullness and pharyngeal disturbances. The otolaryngological examination showed PPS pathology that was confirmed by radiological images. Surgical excision by transcervical-transparotid approach was performed followed by uncomplicated healing with no recurrence in one year. The histological examination reported a pleomorphic parotid adenoma. The authors provide a discussion of the etiopathogenesis, diagnosis and treatment of this type of lesions. This clinical manuscript may shed light on the importance of a complete otolaryngological examination in a patient with unspecific symptoms considering that the PPS is a complex anatomic region and its pathology can easily go unnoticed.
\end{abstract}

Key words: Parapharyngeal space, pleomorphic adenoma, parotid, otic fullness.

1 Médicos del Servicio de Otorrinolaringología, Hospital General Universitario de Castelló, España.

Recibido el 11 de enero, 2018. Aceptado el 15 de marzo, 2018. 


\section{INTRODUCCIÓN}

Los tumores del espacio parafaríngeo (EP) son infrecuentes. Suponen el $0,5 \%-1 \%$ de las neoplasias de cabeza y cuello $0^{1,2}$. Ochenta por ciento son benignos ${ }^{1,2}$ y $40 \%$ corresponden a tumores de glándulas salivales, seguidos de tumores neurogénicos y de las adenopatías ${ }^{3}$. Presentamos el caso de una tumoración de gran tamaño localizada en el espacio parafaríngeo izquierdo así como una revisión de la etiopatogenia, diagnóstico y tratamiento de estas lesiones.

\section{CASO CLÍNICO}

Paciente mujer de 52 años sin antecedentes de interés que acude por presentar sensación de taponamiento de oído izquierdo y molestias faríngeas de 3 meses de evolución. En la exploración se evidencia protrusión de la pared lateral faríngea izquierda y paladar blando hacia línea media. Resto de exploración otorrinolaringológica y palpación cervical y parotídea sin alteraciones. Se realiza una tomografía computarizada (TC) cervical con contraste que muestra una masa en el espacio parafaríngeo preestiloideo izquierdo, bien delimitada, con presencia de vasos en su interior, pero hipodensa y homogénea. Mide $53 \times 30 \times 41 \mathrm{~mm}$. Produce desplazamiento posterolateral de los vasos yugular y carotídeo y anteromedial de la grasa parafaríngea, desplaza la naso y orofaringe medialmente. No se observa plano de separación con la glándula parótida. Se completa el estudio con resonancia magnética (RM), la lesión es hipointensa en T1 e hiperintensa en T2, con una cápsula hipointensa. Dicha masa no es infiltrativa (Figura 1).

Se plantea tratamiento quirúrgico, mediante un abordaje cérvico-parotídeo se realiza parotidectomía total izquierda con preservación del nervio facial, y exéresis de la tumoración (Figura 2). La paciente no presentó complicación alguna. El
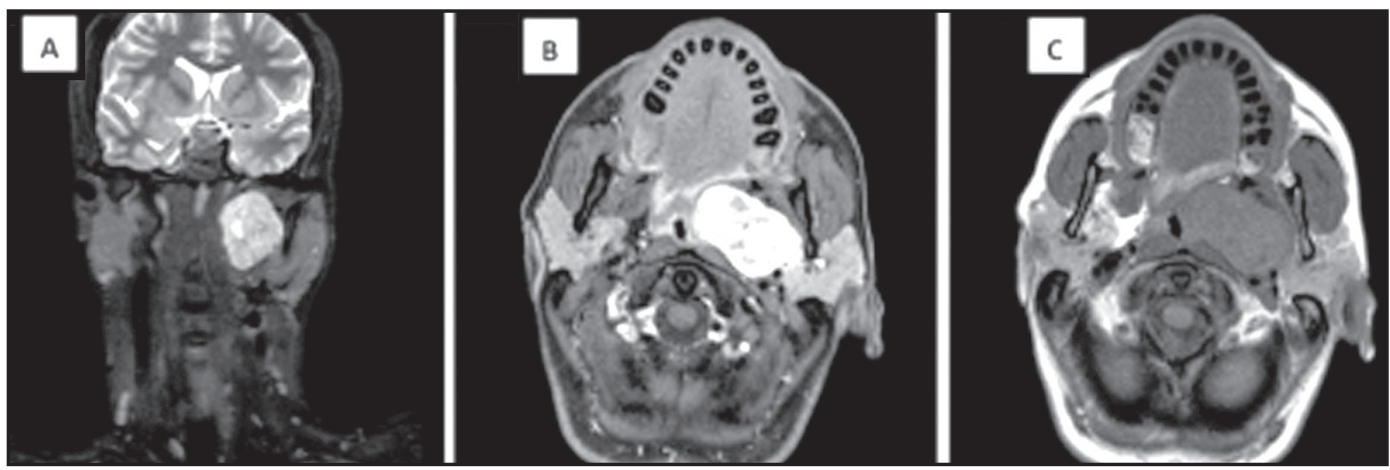

Figura 1. Resonancia magnética. A: Coronal T2, B: Axial T2, masa hiperintensa. C: axial T1: masa hipointensa.

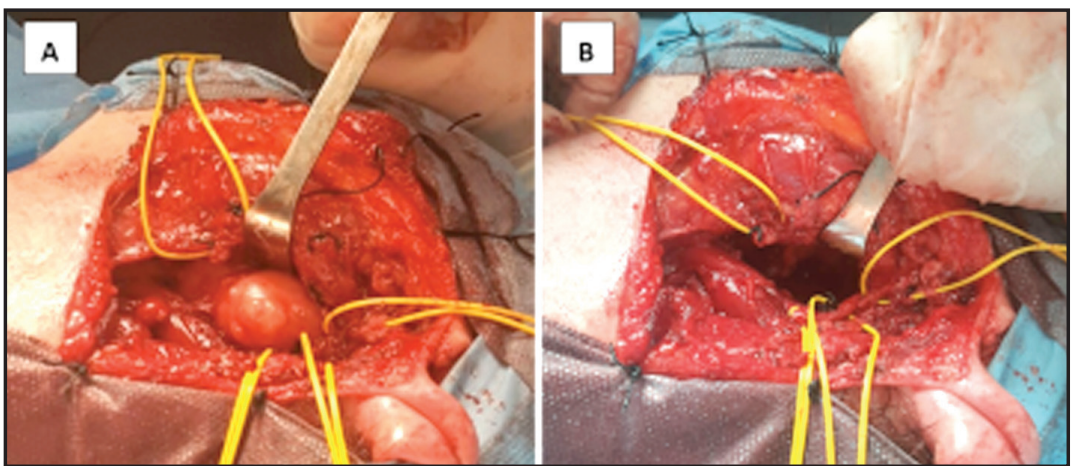

Figura 2. Abordaje cervicoparotídeo. A. Exposición de la tumoración tras disección del nervio facial, B. Cavidad en espacio parafaríngeo izquierdo tras extracción de la tumoración. 
estudio histológico de la pieza extirpada informó de adenoma pleomorfo.

\section{DISCUSIÓN}

El EP es una región anatómica virtual con morfología de pirámide invertida que se extiende desde la base del cráneo hasta el hueso hioides. El límite superior la forma la región petrotimpánica del hueso temporal, y el límite inferior el asta mayor del hueso hioides ${ }^{3,4}$. Si trazamos una línea imaginaria que conecta la apófisis estiloides y el músculo tensor del velo del paladar ${ }^{3,5}$, podemos dividir el EP en espacio retroestiloideo y espacio preestiloideo. El espacio preestiloideo contiene grasa, glándulas salivales menores, ramas de la división mandibular del nervio trigémino, arteria maxilar interna, arteria faríngea ascendente y plexo venoso farínge $0^{6}$. El espacio retroestiloideo contiene los elementos vasculonerviosos principales del cuello: arteria carótida interna, vena yugular interna, ganglios linfáticos, pares craneales IX, X, XI, XII y cadena simpática cervical. Conociendo el contenido de estos espacios podemos sospechar el origen de una tumoración que afecte al EP. Según algunos autores el $75 \%-87 \%$ se trata de lesiones benignas ${ }^{7,8}$. En nuestro caso la localización de la lesión en el espacio preestiloideo, así como la ausencia de un plano graso de separación con la glándula parótida, planteaba como diagnóstico de sospecha inicial un adenoma pleomorfo del lóbulo profundo parotídeo y como segunda opción un neurofibroma o schwanoma.

La mayoría de los tumores ubicados en esta región tienen una evolución indolente y son diagnosticados de forma casual en un estudio radiológico. En el caso que presentamos el tumor tuvo un curso silente hasta que su tamaño fue de 5 $\mathrm{cm}$. La paciente presentó una sintomatología leve e inespecífica (molestias faríngeas y plenitud ótica) de meses de evolución y fue la exploración orofaríngea la que hizo sospechar del diagnóstico de tumoración de EP.

Su localización limita la correcta exploración mediante examen físico por lo que las pruebas de imagen son fundamentales en la valoración de este espacio. La RM con contraste es el método de elección, considerándose la TC con contraste un buen método para los casos en que se precise la valoración ósea o bien en centros donde la accesibilidad a la RM sea menor ${ }^{3}$. Este compartimiento tiene un alto contenido en grasa, y el patrón de desplazamiento de la misma nos podrá orientar sobre el compartimiento afectado. Así pues, en nuestro caso, había un desplazamiento posterolateral de la arteria carótida interna y carótida externa y un desplazamiento medial de la pared lateral del espacio mucoso naso-orofaríngeo, hecho que traducía una localización en el espacio preestiloideo, sin observarse signos de infiltración de estructuras adyacentes. La información que nos aportan las técnicas de imagen es crucial para poder determinar la extensión de la lesión y planificar de este modo el abordaje quirúrgico ${ }^{1,9}$.

En el caso que presentamos no se realizó PAAF previo a la cirugía, dada la alta sospecha de neoformación de características benignas y probable origen parotídeo. La PAAF de tumores del EP no presenta un alto rendimiento en el diagnóstico de tumores pero sí se guía por TC su valor predictivo postivo es del $75 \%-90 \%{ }^{3}$.

El tratamiento de elección es quirúrgico ${ }^{9}$. Se han descrito muchos abordajes quirúrgicos, lo que representa un signo en la dificultad para acceder a esta área anatómica. El tipo de abordaje dependerá del tamaño y localización de la lesión, la relación con los grandes vasos y la sospecha 0 no de malignidad. Conviene tener presente que en la mayoría de los casos se trata de neoplasias benignas, por lo que la cirugía debe conllevar una mínima morbilidad. En la literatura se han descrito diferentes vías de acceso: transoral, transmandibular, acceso de parotidectomía y vía de cervicotomía. El primero no es recomendable porque, aunque se consigue un acceso directo a la lesión, no permite un adecuado control de los vasos y nervios. La vía transmandibular es relativamente excepcional. En nuestro caso se eligió la vía combinada transparotídea-transcervical con propulsión de la mandíbula hacia delante y disección digital del tumor, por su gran tamaño y tipo reloj de arena y su localización en el polo profundo de la glándula parótida, ya que de esta forma se conseguía una exéresis adecuada y completa de la lesión con 
un correcto control sobre las estructuras anatómicas circundantes.

La importancia del caso que hemos expuesto reside en el hecho que la paciente debutó con clínica inespecífica de plenitud ótica y molestias fa-

\section{BIBLIOGRAFÍA}

1. Rodríguez-Ciurana J, Rodado C, Sáez M, Bassas C. Giant parotid pleomorpic adenoma involving the parapharyngeal space: Report of a case. J Oral Maxillofac Surg 2000; 58: 1184-7.

2. Olsen KD, McCaffrey T V. Tumors and Surgery of the Parapharyngeal Space. Laryngoscope 1994; 104: 1-28.

3. Cabezas L, Cardemil F, Cabezas L. Tumores del espacio parafaríngeo. Revisión del tema. Rev Otorrinolaringol Cir Cabeza Cuello 2015; 75: 187-94.

4. Suárez-Fente V, Llorente-pendás JL, Gómez-martínez J, García-gonzález LA, Suárez-Nieto Fl-álvarez C. Tumores primarios del espacio parafaríngeo. Nuestra experiencia en 51 pacientes. Acta Otorrinolaringol Esp 2017; 60: 19-24.

5. Kanzaki S, Nameki H. Standardised method ríngeas, y la exploración orofaríngea fue crucial para sospechar patología del EP. Así pues, esta es una entidad que el otorrinolaringólogo debe tener siempre presente y no debemos olvidar realizar siempre una exploración otorrinolaringológica completa. of selecting surgical approaches to benign parapharyngeal space tumours, based on preoperative images. J Laryngol Otol 2008; 122: 628-34.

6. Álvarez de Eulate Santacara L, Zabala Landa R, Corta Gomez I, Ruiz Morin B, Eguidazu Elosura I, Del Cura Rodríguez J. Lesiones del espacio parafaríngeo: Hallazgos de imagen. SERAM 2012 2012; 1-17.

7. Acosta L, Montalvao P, Magalhaes M. Tumores del espacio parafaríngeo. Nuestra experiencia-IPO Francisco Gentl-Lisboa. Acta Otorrinolaringol Esp 2002; 53: 485-90.

8. Khafif A, Segev Y, Kaplan DM, Gil Z, Fliss DM. Surgical management of parapharyngeal space tumors: A 10-year review. Otolaryngol Head Neck Surg 2005; 132: 401-6.

9. Carrau RL, Myers EN, Johnson JT. Management of tumors arising in the parapharyngeal space. Laryngoscope 1990; 100: 583-9. 